\title{
Applying a Preconditioning Technique to the Euler Equations to Accelerate the Convergence Rate for Low-Speed Flows
}

\author{
Ana A. G. Maia ${ }^{1}$, Janaina F. Silva ${ }^{2}$, Jesuíno T. Tomita ${ }^{3}$, Cleverson Bringhenti ${ }^{4}$ \\ ${ }^{1}$ Aeronautics Institute of Technology \\ Pç. Mal. Eduardo Gomes, 50, São José dos Campos, Brazil \\ ana.adalgiza.eng@ hotmail.com; janaina@ita.br \\ ${ }^{2}$ Aeronautics Institute of Technology \\ Pç. Mal. Eduardo Gomes, 50, São José dos Campos, Brazil \\ jtakachi@ita.br; cleverson@ita.br
}

\begin{abstract}
In an effort to ensure the robustness and numerical stability of a three-dimensional explicit compressible code for all speed flows, a preconditioning technique was implemented. The code solves Euler steady-state equations into a three-dimensional flow. Local preconditioning was implemented due to their accuracy in predicting lift and drag forces on mixed flows. However, for low speed flows near stagnation points numerical perturbations are amplified, generating a loss in the convergence rate, code accuracy and robustness. Aiming to improve the preconditioning accuracy and convergence rate suggested a new limit to the preconditioning sensor based on the flow pressure. Numerical simulations of a subsonic flow over a cylinder showed a faster convergence rate when the preconditioning technique was implemented.
\end{abstract}

Keywords: CFD, Preconditioning, Eigenvalues, Mach number, All Speed.

\section{Nomenclature}

$Q$

$E_{e}, F_{e}, G_{e}$
$A, B, C$

Vector of conservative variables

Flux vectors

$I$ Jacobian matrix of flux vectors

$u, v, w$ Identity matrix

$\rho$

$p$

$T$

a

E

$e$

$\gamma$

$n_{x}, n_{y}, n_{z}$

Velocity in Cartesian coordinates

Density

Pressure

Temperature

Sound speed

Total stagnation energy

Internal energy

$a$

$\beta$

Ratio of specific heats

Normal vector unit in Cartesian coordinates

Free parameter constant

Preconditioning sensor

$M \quad$ Mach number

\section{Introduction}

Advances in Computational Fluid Dynamics (CFD) in the last two decades increased the accuracy of numerical solutions. Difficulties to solve compressible equations for mixed speed regimes were observed by several authors [1-7]. The preconditioning techniques were created to ensure the robustness and stability of compressible codes for all speed flows.

The first author which studied the stiffness in the convergence to solve compressible codes for low Mach number flows was Chorin [1]. The author demonstrated that the low convergence rate was caused by the large disparity between the acoustic wave speed and the waves propagating at the fluid speed. Aiming to solve that Chorin created the artificial compressibility 
technique which consists in added the pressure time derivative in the continuity equation acquiring a symmetric hyperbolic system to ensure the solution convergence, however, the time accuracy was lost.

Following the proposed by Chorin [1], Choi and Merkle [2] and Turkel [3] modified the artificial compressibility technique, adding the pressure time derivative in the momentum equations, that was named preconditioning. With that, the system condition number is altered to be independent of the flow Mach number ensuring the numerical solution convergence and preserving the solution accuracy.

As the preconditioning matrix multiplies the spatial operator are modified the solution eigenvalues and eigenvectors. More studies of preconditioning techniques are looking to change the eigenvalues to obtain a symmetric system reducing the spread of the characteristics speed [8-12] then the preconditioning matrix should be chosen for each case. Turkel [8] and Allmaras [9] demonstrated that the code based on the primitive variables provides a fast convergence rate and decreases the numerical instabilities for low-speed flows. Using this idea, the authors proposed a new preconditioning matrix $(\mathrm{P})$ which also modifies the solution dependent variables, getting improvements in the code robustness decreasing the stiffness in the convergence rate [10-12].

Darmofal and Schmid [13] applied three local preconditioners of Lee [6], Turkel [8], and Allmaras [9] in the Euler equations. The preconditioning systems of van Leer and Turkel demonstrated a transient perturbation growth near stagnation points, due to the eigenvectors non-orthogonality at low Mach numbers. Although, the block-Jacobi preconditioner [13] does not amplify the perturbations the preconditioner does not accelerate the convergence due to the long wavelength mode when the Mach number goes to zero. Then, modifications are suggested in the van Leer and Turkel's preconditioners and in their sensor $(\beta)$, as a function of the flow Mach number, improving the robustness of the upwind finite volume code.

In the work of Turkel, Vatsa, and Radespiel [14] pointed out problems in calculating lift and drag coefficients in wing profiles for flows with Mach numbers lower than 0.1 to a multigrid algorithm. To solve that, they created a new preconditioning matrix based on Turkel [8] for compressible codes and developed a new preconditioning sensor $(\beta)$ with a simple cut-off, which calculates this sensor for all control volumes. Predictions of lift and drag coefficients are improved as the convergence rate for mixed speed flows.

Darmofal and Siu [15] made a Fourier analysis in the Turkel's preconditioning [8] in two-dimensional Euler equations through a multigrid algorithm. They established a new limit to the preconditioning sensor to ensure the balance between the eigenvalues and the flow speed, and modify the upwind flux function in the preconditioning. As a result, it decreases the numerical amplifications near stagnation points improving the code robustness.

In this paper, the preconditioning technique was applied in a three-dimensional explicit compressible code developed by Tomita [16] to solve Euler equations. The preconditioning of Turkel, Vatsa, and Radespiel [14] was applied due to the ability to solve flows with mixed speed regimes. As local preconditioners when the free stream Mach number is close to zero could be shown near stagnation points or in regions with the significant low-speed flow, inaccurate solutions due to the transient amplification of perturbations. Concerning a more robust preconditioning technique was applied a new limit to the preconditioning sensor and an explicit flux function proposed by Darmofal and Siu [15]. The numerical simulations for the inviscid flow are done in a flow over at cylinder. First, in the compressible case, the flow Mach number is set 0.17 and, next to the incompressible case the flow Mach number is set 0.05 . The changes in the preconditioning technique demonstrated improvements in the convergence rate for low Mach number flows.

\section{Mathematical Formulation}

Eq. (1) shows a vector form of the 3D Euler equations,

$$
\frac{\partial \vec{Q}}{\partial t}=-\left[\frac{\partial \overrightarrow{E_{e}}}{\partial x}+\frac{\partial \overrightarrow{F_{e}}}{\partial y}+\frac{\partial \overrightarrow{G_{e}}}{\partial z}\right],
$$

Where, $\vec{Q}$ is the vector of conservative variables $\vec{E}, \vec{F}$, and $\vec{G}$ are the flux vectors and, the subscript $e$ indicates the inviscid part (Euler), 


$$
\vec{Q}=\left[\begin{array}{c}
\rho \\
\rho u \\
\rho v \\
\rho w \\
\rho E
\end{array}\right], \overrightarrow{E_{e}}=\left[\begin{array}{c}
\rho u \\
\rho u^{2}+p \\
\rho u v \\
\rho u w \\
(\rho E+p) u
\end{array}\right], \overrightarrow{F_{e}}=\left[\begin{array}{c}
\rho v \\
\rho v u \\
\rho v^{2}+p \\
\rho v w \\
(\rho E+p) v
\end{array}\right], \overrightarrow{G_{e}}=\left[\begin{array}{c}
\rho w \\
\rho w u \\
\rho w v \\
\rho w^{2}+p \\
(\rho E+p) w
\end{array}\right]
$$

The variables are dimensionless, $\rho$ is the flow density, $u, v$, and $w$ are velocity in Cartesian coordinates, $p$ is static pressure, and $E$ is total stagnation energy defined by Eq. (2),

$$
\mathrm{E}=\mathrm{e}+\frac{1}{2}\left(u^{2}+v^{2}+w^{2}\right)
$$

Writing the normal flux of the $E, F$, and $G$ vectors in function of the Jacobians $A=\partial E_{e} / \partial x, B=\partial F_{e} / \partial y$, and $C=$ $\partial G_{e} / \partial z$ resulted in,

$$
D_{n}=\mathbf{A} n_{x}+\mathbf{B} n_{y}+\mathbf{C} n_{z}
$$

where, $n_{x}, n_{y}$, and $n_{z}$, correspond to the normal vector $\boldsymbol{n}$ defined as $\boldsymbol{n}=\left[\begin{array}{lll}n_{x} & n_{y} & n_{z}\end{array}\right]^{T}$. The matrix $D_{n}$ is,

$$
D_{n}=\left(\begin{array}{ccccc}
0 & n_{x} & n_{y} & n_{z} & 0 \\
\theta / 2 q_{n}{ }^{2} n_{x}-u q_{n} & u n_{x}-\theta u n_{x}+q_{n} & u n_{y}-\theta v n_{y} & u n_{z}-\theta w n_{z} & \theta n_{x} \\
\theta / 2 q_{n}{ }^{2} n_{y}-v q_{n} & v n_{x}-\theta u n_{y} & v n_{y}-\theta v n_{y}+q_{n} & v n_{z}-\theta w n_{y} & \theta n_{y} \\
\theta / 2 q_{n}{ }^{2} n_{z}-w q_{n} & w n_{x}-\theta u n_{z} & w n_{y}-\theta v n_{z} & w n_{z}-\theta w n_{z}+q_{n} & \theta n_{z} \\
\left(\theta / 2 q_{n}{ }^{2}-H\right) & H n_{x}-\theta u q_{n} & H n_{y}-\theta v q_{n} & H n_{z}-\theta w q_{n} & \gamma q_{n}
\end{array}\right) .
$$

Where, $\theta=\gamma-1$ and $H=p / \rho . q$ and $q_{n}$ are,

$$
\begin{gathered}
q_{n}=\mathrm{u} n_{x}+v n_{y}+w n_{z} \\
q_{n}^{2}=u^{2}+v^{2}+w^{2}
\end{gathered}
$$

Compressible codes for low speed flows need to apply the primitive variables to increase the convergence rate. Aiming a better convergence rate the resultant matrix is composed by the Jacobian matrix $(\partial q / \partial Q)$ to change the variables and the preconditioning matrix $(P)$ to change the solution eigenvalues. Multiplying the matrix $D_{n}$ by the resultant matrix $(\Gamma)$ and substituting Eq. (3) in Eq. (1),

$$
\frac{\partial \vec{q}}{\partial t}=-\mathrm{P} D_{n}(Q)
$$

Where, $P=\Gamma \partial Q / \partial q$. The matrix $\Gamma$ is, 


$$
\Gamma=\left(\begin{array}{ccccc}
\beta^{2} & 0 & 0 & 0 & 0 \\
-\frac{(\alpha+1) u}{\rho} & 1 / \rho & 0 & 0 & 0 \\
-\frac{(\alpha+1) v}{\rho} & 0 & 1 / \rho & 0 & 0 \\
-\frac{(\alpha+1) w}{\rho} & 0 & 0 & 1 / \rho & 0 \\
\widehat{T}\left[\beta^{2}+\frac{q_{n}^{2}}{2}-\frac{a^{2}}{\gamma-1}\right] & \widehat{T} u & \widehat{T} v & \widehat{T} w & \widehat{T}
\end{array}\right)
$$

$\widehat{T}$ is the dimensionless temperature,

$$
\widehat{T}=\frac{(\gamma-1) T}{\gamma p}
$$

$a$ is the sound speed, $\alpha$ is a free parameter constant set in the range $0 \leq \alpha \leq 1$, and $\beta$ is the preconditioning sensor established by Turkel, Vatsa and Radespiel [14] is scaled with the speed flow, ensuring the proportionality of the solution eigenvalue and the flow speed, then,

$$
\beta^{2}=\min \left\{\max \left[K_{1}\left(u^{2}+v^{2}+w^{2}\right)\left(1+\frac{1-M_{0}^{2}}{M_{0}^{4}}\right), K_{2}^{2}\left(u^{2}+v^{2}+w^{2}\right)\right], a^{2}\right\}
$$

With $K_{1}$ between 1.0 and 1.1 and $K_{2}$ is between 0.4 and 1.0. The value of $\beta$ is calculated for each cell of the control volume. Analysing the Eq. (11), for high Mach numbers $\beta^{2}=a^{2}$ then $\Gamma=I$ returning to the original code.

\subsection{A new limit for the preconditioning sensor}

The preconditioning method of the Turkel, Vatsa, and Radespiel [14] for low speed flows showed transient amplifications near stagnation points which causes a robustness loss, these amplifications were shown by Darmofal and Schimid [13] then, some changes were proposed to improve the preconditioner performance.

Darmofal and Siu [15] did a Fourier analysis showing the transient amplification growth to the local preconditioners when the preconditioning sensor goes to zero. Therefore, the authors suggested a limit to the preconditioning sensor based on the pressure to avoid that possibility. The $\beta_{\text {lim }_{\text {new }}}$ is defined as,

$$
\beta_{\text {limew }}=\frac{\left|p_{R}-p_{L}\right|}{\rho a^{2}}
$$

where, $\mathrm{L}$ and $\mathrm{R}$ are the neighbours in the left and right sides of each face of the control volume. For each cell of the control volume, $\beta_{\text {cell }}^{2}$ is,

$$
\beta_{\text {cell }}^{2}=\max _{k=1}^{k=6}\left(\beta_{\text {face }}^{2}\right)_{k}
$$

Where, $\beta_{\text {face }}^{2}$ is defined as,

$$
\beta_{\text {face }}^{2}=\min \left[1, \max \left(\beta_{L}^{2}, \beta_{R}^{2}, \beta_{\text {lim new }}^{2}\right)\right] .
$$

The flux of the preconditioning sensor for each cell of the control volume is calculated by the Eq. (12), 


$$
\beta_{\text {flux }}^{2}=\max \left(\beta_{\text {cell }}^{2}, \beta_{\text {cell }}^{2}\right)
$$

Darmorfal and Siu [15] also redefined $\alpha$ as a function of the Mach cut off $\left(M_{0}\right)$,

$$
\alpha=\frac{\left(1-M_{0}^{2}\right)}{M_{0}^{2}} .
$$

For low speed flows the eigenvalues are small causing a decrease in the time step which decreases the convergence rate. Then, Turkel, Vatsa and Radespiel [14] suggested the Eq. (14) to calculate the time step to the preconditioning cells.

$$
\Delta t=\frac{C F L}{\frac{1}{2}\left[\left|q_{n}\right|\left(1-\beta^{2}\right)+\sqrt{q^{2}\left(1-\beta^{2}\right)+4 \beta^{2} a^{2}}\right]} \Delta x
$$

\section{Numerical Simulation}

Tomita [16] developed a three-dimensional compressible code which solves Euler and Navier-Stokes equations. The spatial discretization is done applying the cell-centered finite volume method. The centered scheme discretized the convective terms, also due to the numerical instabilities caused by the centered scheme the artificial dissipation was added based in the work of the Jameson, Schmid, and Turkel [17].

To implement the preconditioning technique in the original code was required the total residual composed by the convective, viscous flux and artificial dissipation [18]. Then, the residual is multiplied by the Jacobian matrix and next to the preconditioning matrix $(\Gamma)$, finally, the five steps Runge-Kutta scheme is applied to make the time integration. Aiming to accelerate the convergence rate is applied to the implicit residual smoothing (IRS) of the Jameson, Schmid, and Turkel [17]. After that, primitive variables were changed to conservative for the next time steps. Lastly, the boundary conditions are applied outside of the flow domain through ghost cells to ensure the correct information in the boundary cells.

The inviscid flow (Euler equations) is tested to a flow over a cylinder submitted to a subsonic flow. First, the Mach number is set as 0.17 , and to test the preconditioning performance the free stream Mach number was decreased to 0.05 . In both cases, the original compressible code (named as original) and the proposed preconditioning (named as Preconditioning), were tested and compared.

\subsection{Flow over a cylinder}

The simulation of a cylinder is done considering its symmetry in the x-direction. Several authors studied the flow past a cylinder in a uniform stream [19] showing two stagnation points, one at the front and another at the back of the cylinder which also produced a stagnation region in the flow downstream. It is considered a cylinder with a diameter of $1 \mathrm{~mm}$, the mesh creation respected the non-reflection conditions in the boundary as established by [20]. A three-dimensional hexahedral mesh with 27,306 nodes, 17,680 volumes and 18,724 2D elements is used in the numerical simulations (Fig. 1). The boundary conditions applied were: velocity inlet, far-field and symmetry. It was set upstream of the cylinder the free-stream Mach number as 0.17 , and the CFL number was 0.1. 


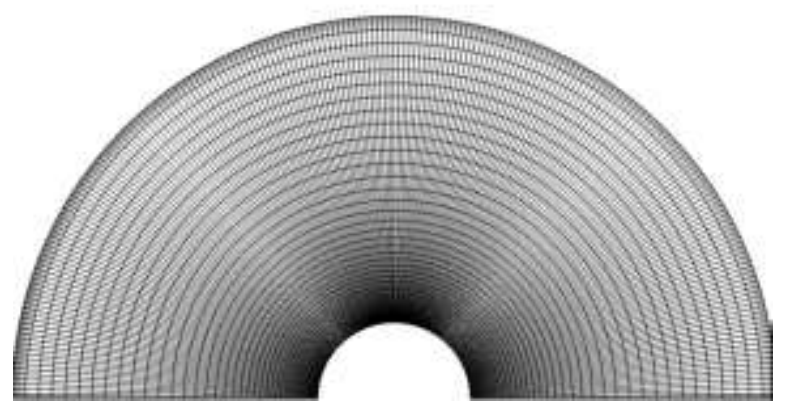

Fig. 1: Sketch of the mesh used to simulate the cylinder.

Figs. 2 and 3 present the density and Mach contours to the original and preconditioning code.

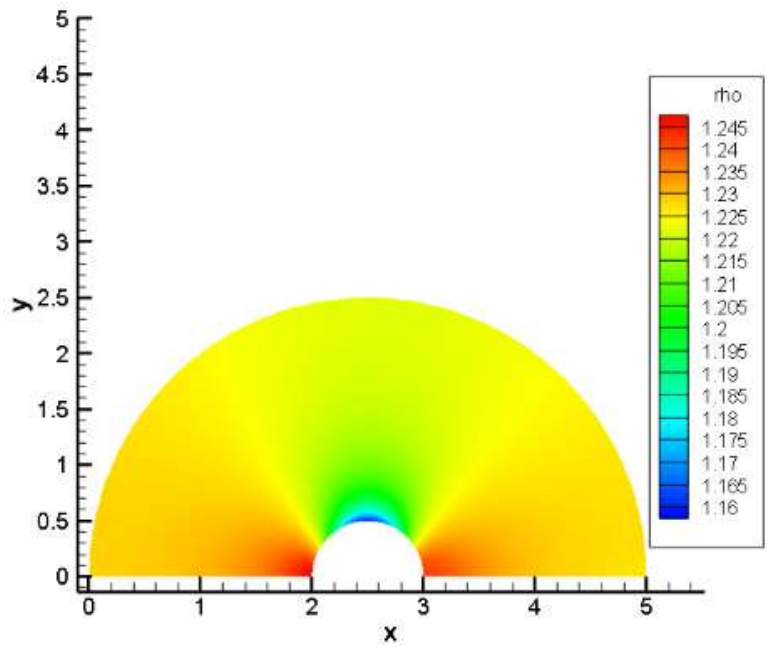

(a) Original.

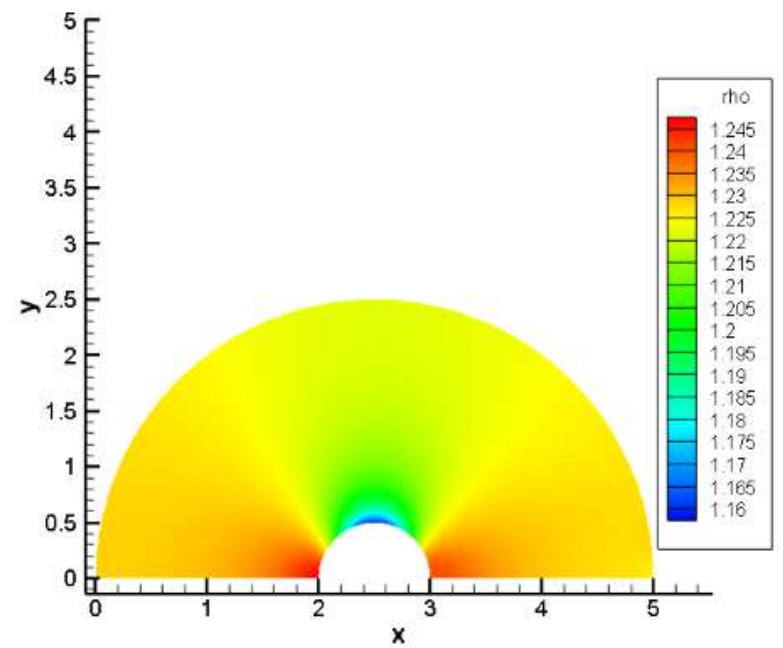

(b)Preconditioning 1.

Fig. 2: Density contours to the original and preconditioning methods $(M=0.17)$.

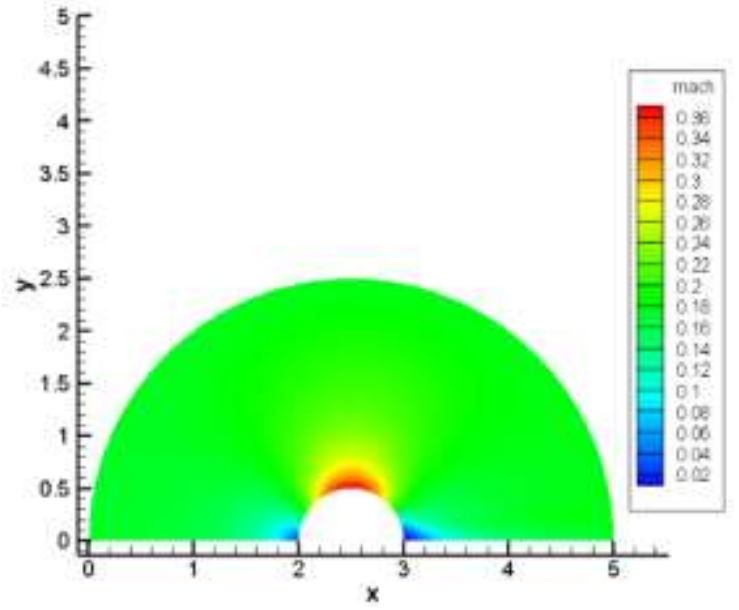

(a) Original.

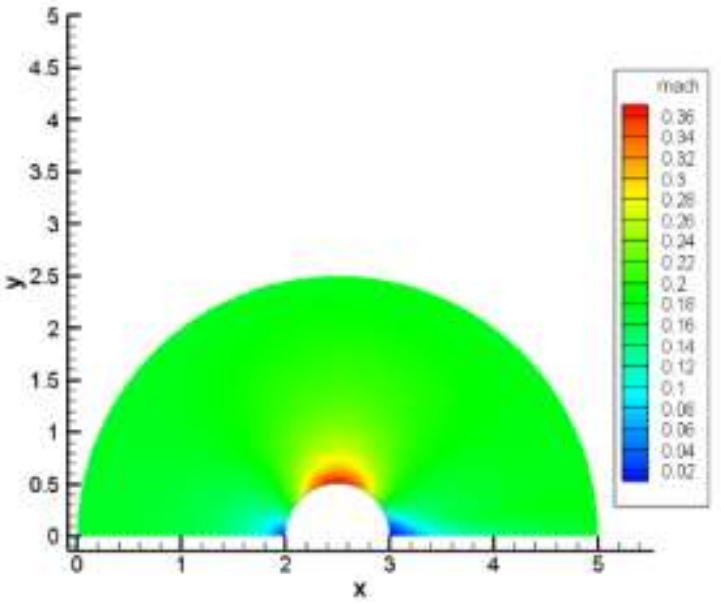

(b)Preconditioning 1.

Fig. 3: Mach contours to the original and preconditioning methods $(M=0.17)$. 
Analysing the Figs. 2 and 3 are shown close solutions for the original and preconditioning methods with the same distribution of density and Mach contours. The highest Mach number localized on the forward surface is 0.36 and is also also showing higher stagnation region in the cylinder downstream. In Fig. 4 are present the distribution of Mach contours with lines to show the flow behaviour close to the cylinder, mainly near the stagnation points, demonstrating a close distribution of both methods without degrading in the solution.

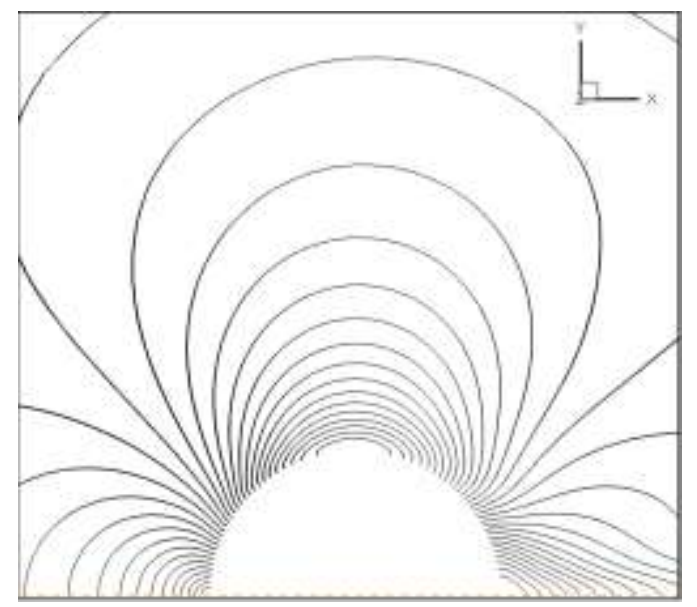

(a)Original

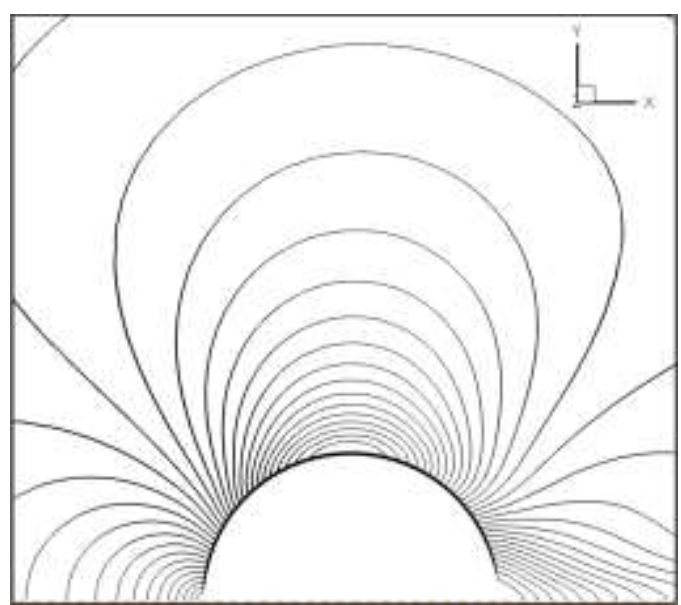

(b)Preconditioning 2.

Fig. 4: Mach contours to the three methods. $M=0.17,30$ equally spaced contours from $M=0.012$ to 0.36 .

The logarithmic residual history of the original and preconditioning methods is shown in Fig. 5.

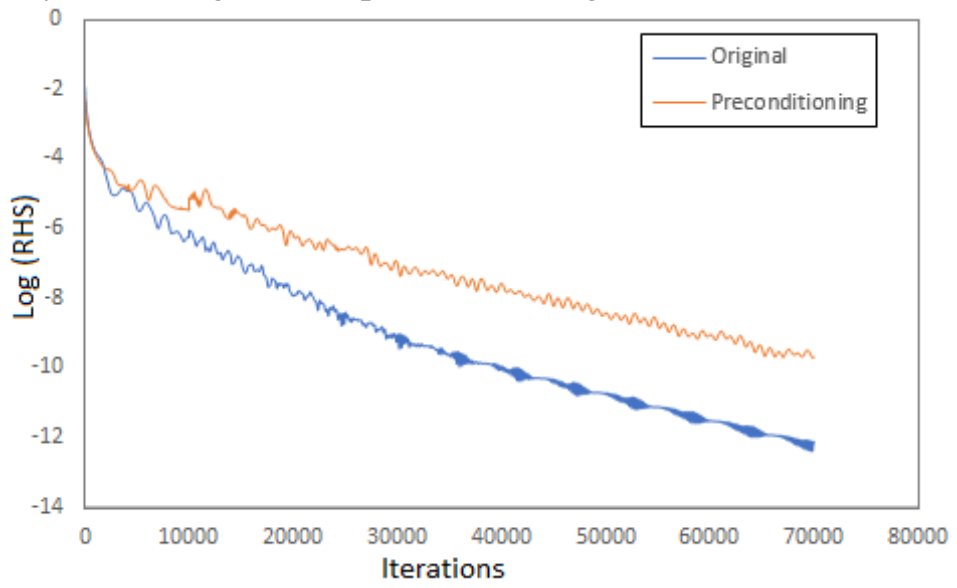

Fig. 5: Log residual histories for a flow over a cylinder.

To demonstrate the preconditioning performance for a low-speed flow, the free-stream Mach number is decreased to 0.05 making the flow essentially incompressible. The original and preconditioning methods were tested. The CFL number adopted was 0.1 . 


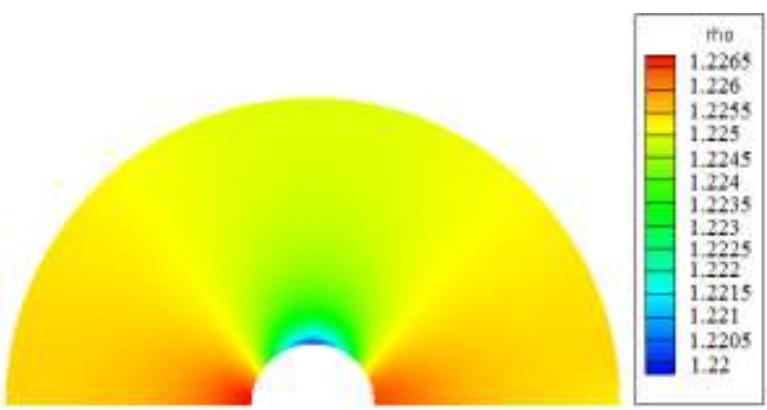

(a) Original.

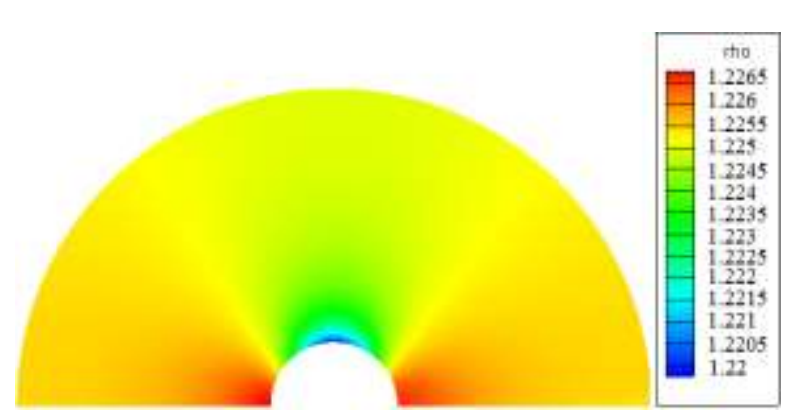

(b) Preconditioning 2.

Fig. 6: Density contours to the three methods $(M=0.05)$.

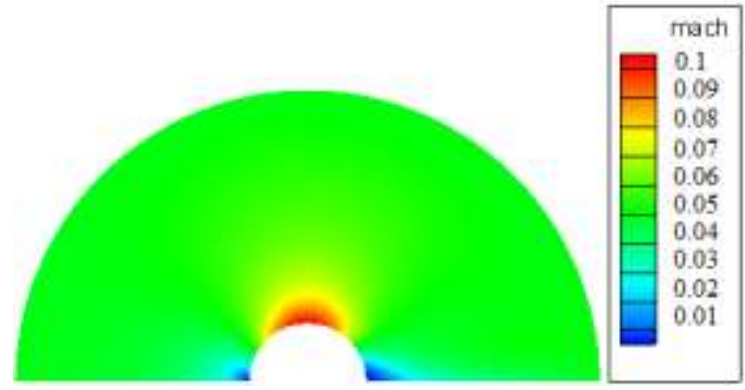

(a) Original.

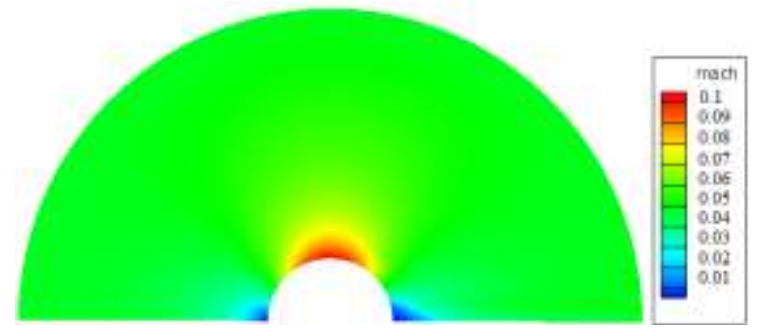

(b) Preconditioning 2.

Fig. 7: Mach contours to the three methods $(M=0.05)$.

The density and Mach contours had close solutions for the original and preconditioning methods. It shows the highest value of Mach number is 0.1 and the stagnation points with lower speed are presented in the cylinder upstream and downstream. Although the original compressible code had accurate solutions for a flow Mach number lower than the incompressible limit $(\mathrm{M}=0.1)$ for more complex cases its accuracy could be loss [16]. Fig. 8 shows the lines of Mach number contour to flow past a cylinder.

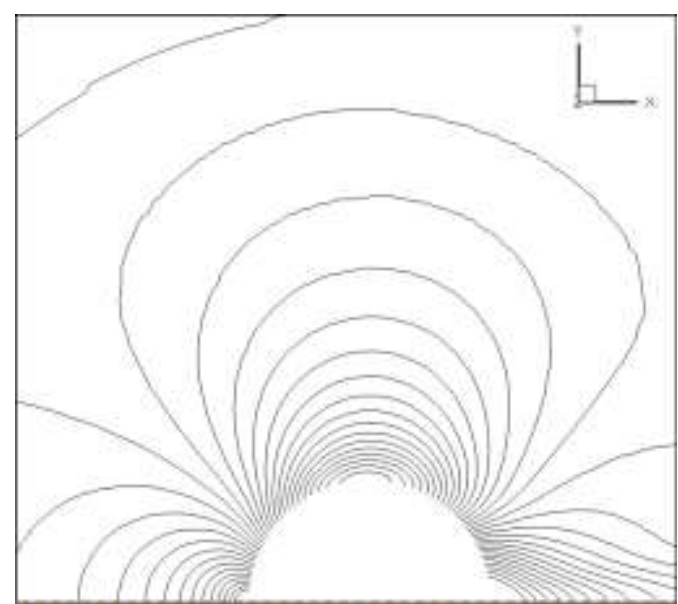

(a) Original.

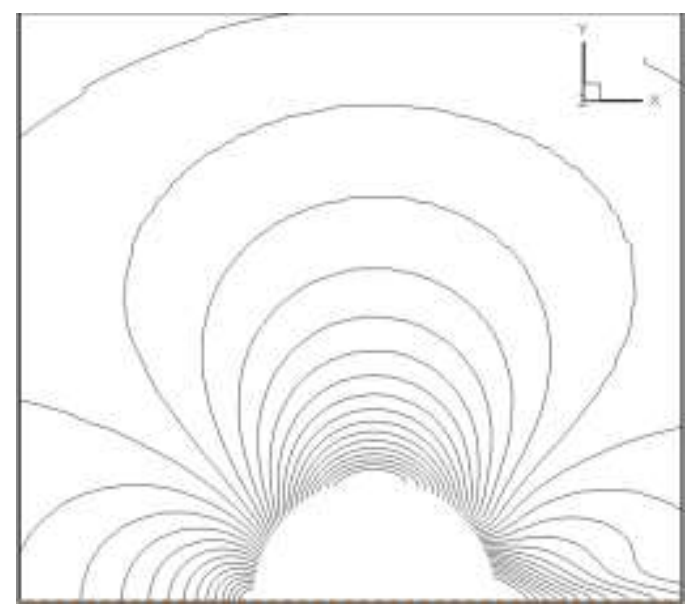

(b) Preconditioning.

Fig. 8: Mach contours to the three methods. $M=0.17,30$ equally spaced contours from $M=0.012$ to 0.36 .

The lines in Fig. 8 show a close distribution of Mach contours over a cylinder for both methods. It is present that the solution has begun to degrade due to the low-speed flow. Fig. 9 presents the logarithm residual histories for original and preconditioning methods. 


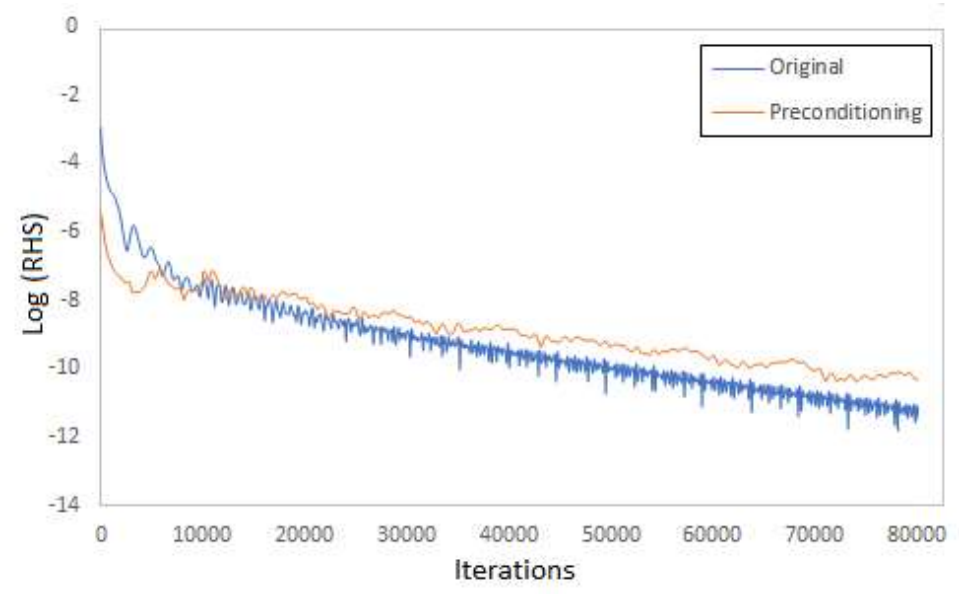

Fig. 9: Log residual histories for a flow over a cylinder.

In Fig. 9 is shown a higher decay to the preconditioning code, with 1110 iterations the preconditioning code is converged and to the 5740 iterations, the original code is converged indicating an accelerating in the convergence rate when the preconditioning method is implemented.

\section{Conclusion}

In the present work, a preconditioning technique was applied to a 3D compressible CFD code. Research into the literature, the local preconditioning method of Turkel, Vatsa, and Radespiel [14] was applied due to the accuracy of predicting the lift and drag coefficients in airfoils. After some tests for low speed flows the preconditioning code showed a loss in the robustness, therefore modifications in the original preconditioning technique were proposed. For that, it is established an explicit flux function and a new limit to the preconditioning sensor based on the work of Darmofal and Siu [16].

For the compressible case with a flow Mach number of 0.17 both methods showed close solutions with the flow Mach number distribution as shown in the literature [19]. Decreasing the flow Mach number to 0.05 the numerical solutions are close for both cases, however, near stagnation points, the solution is beginning to degrade. The preconditioning implemented in the original code increases the convergence rate for low flow Mach number.

All in all, the original code did not lose the solution accuracy for low Mach number flows, however, for complex cases, it does not guarantee the accuracy. Implementing the preconditioning technique in the original compressible code the solution convergence rate is accelerated.

\section{Acknowledgements}

This work was supported by the CNPq (Conselho Nacional de Desenvolvimento Científico e Tecnológico); and CAPES (Coordenação de Aperfeiçoamento de Pessoal de Nível Superior).

\section{References}

[1] A. J. Chorin, "A numerical method for solving incompressible viscous flow problems," J. Comput. Phys., vol. 2, no. 1, pp. 12-26, 1967.

[2] Y. H. Choi and C. L. Merkle, "Application of time-iterative schemes to incompressible flow," J. AIAA, vol. 23, no. 10, pp. 1518-1524, 1985.

[3] E. Turkel, "Preconditioning methods for solving incompressible and low-speed compressible equations," J. Comput. Phys., vol. 72, no. 2, pp. 277-298, 1987.

[4] B. van Leer, W. T. Lee and P. L. Roe, "Characteristic time stepping or local preconditioning of the Euler equations," in 10th Computational Fluid Dynamics Conference, Honolulu, HI, 1991.

[5] D. Lee and B. van Leer, "Progress in Local Preconditioning of the Euler and Navier-Stokes Equations," AIAA Paper, vol. 93, no. 3330, pp. 338-348, 1993. 
[6] C. L. Merkle, S. Venkateswaran and P. E. Buelow, "The relationship between pressure-based and density-based algorithms," in 30th Aerospace Sciences Meeting and Exhibit, Reno, NV, AIAA, 1992.

[7] R. Lohner, C. Yang, J. Cebral, F. Camelli, O. Soto and J. Waltz, "Improving the speed and accuracy of projectiontype incompressible flow solvers," Comp. Methods in Applied Mechanics and Engineering, vol. 195, pp. 3087-3109, 2006.

[8] E. Turkel, "Review of preconditioning methods for fluid dynamics," Applied Numer. Mathemat., vol. 12, pp. 257-284, 1993.

[9] S. R. Allmaras, "Analysis of a local matrix preconditioner for 2-D Navier-Stokes equations," in 11th Computational Fluid Dynamics Conference, Orlando, FL, AIAA, 1993.

[10] Y. Achdou, P. Tallec, F. Nataf and M. Vidrascu, "A domain decomposition preconditioner for an advection-diffusion problem," Comp. Methods in Applied Mechanics and Engineering, vol. 184, pp. 145-170, 2000.

[11] G. Hauke and T. J. R. Hughes, "A comparative study of different sets of variables for solving compressible and incompressible flows," Comp. Methods in Applied Mechanics and Engineering, vol. 153, pp. 1-44, 1998.

[12] M. M. Ginardi, G. Bernardino, M. Vázquez and G. Houzeaux, "Fourier stability analysis and local Courant number of the preconditioned variational multiscale stabilization (P-VMS) for Euler compressible flow," Comp. Methods in Applied Mechanics and Engineering, vol. 301, pp. 28-51, 2016.

[13] D. L. Darmofal and P. J. Schimid, "The importance of eigenvectors for local preconditioners of the Euler equations," J. Comput. Phys., vol. 127, no. 2, pp. 346-362, 1996.

[14] E. Turkel, V. N. Vatsa and R. Radaspiel, "Preconditioning methods for low-speed flows," in 14th Applied Aerodynamics Conference, New Orleans, LA, AIAA, 1996.

[15] D. L. Darmofal and K. Siu, "A robust multigrid algorithm for the Euler equations with local preconditioning and semicoarsening," J. Comput. Phys., vol. 151, no. 2, pp. 728-756, 1999.

[16] J. T. Tomita, "Three-dimensional flow calculations of axial compressors and turbines using CFD techniques," $\mathrm{PhD}$ Thesis, Dept. Turb., ITA, São José dos Campos, SP.

[17] A. Jameson, W. Schimid and E. Turkel, "Numerical solution of the Euler equations by finite volume methods using Runge-Kutta time-stepping schemes," in 14th Fluid and Plasma Dynamics Conference, Palo Alto, CA, 1981, pp. 124.

[18] A. A. G. Maia, "Cálculo de Escoamento em Grades de Turbomáquinas Utilizando a Dinâmica dos Fluidos Computacional Com o Uso de Pré-condicionadores," Master Thesis, Dept. Turb., ITA, São José dos Campos, SP, 2014.

[19] D. Jespersen, T. Pulliam and P. Bunning, "Recent enhancements to overflow," AIAA Paper, pp. 97-0644, 1997.

[20] A. Jameson and D. Mavriplis, "Finite volume solution of the two-dimensional Euler equations on a regular triangular mesh," J. AIAA, vol. 24, no. 4, pp. 611-618, 1986. 